\title{
The Anti-hepatotoxic Effect of Ginseng in Rats: Meta-analysis
}

\author{
Sejeong Kook ${ }^{1}$, Hye-Kyoung Han ${ }^{1,2}$, Gun-Hee Kim ${ }^{1,2}$ and Kiheon Choi ${ }^{3 \#}$ \\ ${ }^{1}$ Plant Resources Research institute, Duksung Women's University, Seoul, 132-714, South Korea \\ ${ }^{2}$ Department of Food and Nutrition, Duksung Women's University, Seoul, 132-714, South Korea \\ ${ }^{3}$ Department of Statistics, Duksung Women's University, Seoul, 132-714, South Korea
}

(Received April 30, 2008; Accepted June 14, 2008)

\begin{abstract}
The aim of this meta-analysis was to systematically investigate the anti-hepatotoxic effect of ginseng in rats induced toxicity which damage to liver. Primary researches were gained on the ScienceDirect database, the DBpia, and the KISS, and the data about the effect factors in plasma and in enzyme were listed as many as possible. The effect factors were alanine transaminase (ALT), aspartate transaminase (AST), liver aminopyrine $\mathrm{N}$-demethylase (AD), liver aniline hydroxylase (AH), liver 3,4-Methylenedioxyamphetamine (liver MDA), cytochrome P450 (P450), serum alkaline phosphatase (ALP), serum lactate dehydrogenase (LDH), cytochrome b5 (Cyto b5), glutathione reductase (GR), Liver glutathione $S$-transferase (GST), liver glutamyltransferase (GT), Liver ( $\gamma$-GCS), serum liver 3,4-Methylenedioxyamphetamine (serum MDA), serum sorbitol dehydrogenase (SDH), serum total protein (TP), serum $\gamma$-glutamyltransferase $(\gamma$-GT). To investigate the effect of ginseng, the mean difference (MD) between the group of rats induced by toxicity (RH) and the group of rats induced by toxicity with ginseng (RHG) were combined, and the significance of MDs were tested. The combined MDs were checked the biases caused by heterogeneity among studies and the publication biases, and adjusted by using random effect model and trim and fill method, respectively. The effect about ALT, AST, ALP, LDH, SDH, TP and $\gamma$-GT in plasma factors were significant, and about AD, liver MDA, P450, Cyto b5, GR, GST, GT and $\gamma$-GCS in enzyme factors were significant. The treatment with ginseng supplementation was significantly effected on plasma and enzyme factors of damaged-rats.
\end{abstract}

Key words : hepatoprotective, galactosamine, meta-analysis, publication bias

\section{INTRODUCTION}

Drug-induced liver injury is a common form of adverse drug reaction and accounts for more than $50 \%$ of cases of acute liver failure in the United States ${ }^{1)}$. More than 900 drugs, toxins, and herbs have been reported to cause liver injury, and drugs account for $20-40 \%$ of all instances of fulminant hepatic failure. Drug-induced hepatic injury is the most common reason cited for withdrawal of an approved drug. The manifestations of drug-induced hepatotoxicity are highly variable, ranging from asymptomatic elevation of liver enzymes to fulminant hepatic failure ${ }^{2)}$. Medicinal herbs and extracts prepared from them are widely used in the treatment of liver diseases like hepatitis, cirrhosis, and loss of appetite ${ }^{3)}$. A number of recent reviews have focused on the adverse effects of herbal products ${ }^{4}$.

\footnotetext{
\#To whom correspondence should be addressed.

(Tel) +82-2-901-8332; (Fax) +82-2-901-8661

(E-mail) khchoi@duksung.ac.kr
}

The herbal remedies referred to as ginseng are derived from the roots of several plants. One of the most commonly used and researched of the ginsengs is Panax ginseng, also called Asian or Korean ginseng. The main active components of Panax ginseng are ginsenosides, which have been shown to have a variety of beneficial effects, including anti-inflammatory, antioxidant, and anticancer effects. Overall, Panax ginseng appears to be well tolerated, although caution is advised about concomitant use with some pharmaceuticals, such as warfarin, oral hypoglycemic agents, insulin, and phenelzine. Panax ginseng does not appear to enhance physical performance. Products with a standardized ginsenoside concentration are available ${ }^{5)}$. The main active agents in Panax ginseng are ginsenosides, which are triterpene saponins. The majority of published research on the medicinal activity of Panax ginseng has focused on ginsenosides ${ }^{6}$. Panax ginseng is well known to enhance the release of nitric oxide from endothelial cells of the rat aorta and to reduce blood pressure in animals ${ }^{7}$. As the studies about anti-dia- 
betic effect of ginseng are increased, it is necessary that the results are combined and summarized.

Meta-analysis is a tool for summarizing the results of studies related research hypotheses. Meta analysis has three steps, deciding the effect measure for difference between groups, summarizing the effect measure and detecting publication bias. The interested measure in meta-analysis is the standardized measure of effect size extracted from the primary research such as the standardized mean difference, the risk difference, and an odds ratio. After deciding the effect measure, the effect measures are combined and summarized under assumed model. It extracts more convincing conclusions than that obtained from individual studies, because of its capability of reducing the random errors in the assessment of treatment. In small number of studies, the publication bias easily occurs. Publication bias occurs when the summarized estimate are calculated only published researches. Significant results are more likely to be submitted or accepted than not significant one ${ }^{8,9)}$.

The purpose of this study was to summarize the factors related the anti-hepatotoxic effects of ginseng on induced rats by toxicity.

\section{METHODS}

\section{Preparation of dataset for meta-analysis}

The studies used in this meta-analysis were searched on the ScienceDirect in english and the DBpia database and the KISS (Koreanstudies Information Service System) in korean. The search keywords used were ginseng, panax or hepatotoxicity. The research was limited to experimental rat studies. 5 primary studies were gained, and there were control group and treated group in each primary study. The RH was a group of rats induced by hepatotoxicity, such as carbon tetrachloride or 2,3,7,8-tetrachlorodibenzop-dioxin, and the RHG was a group of rats induced by hepatotoxicity with ginseng. The factors selected to research the effect of Ginseng were the levels alanine transaminase (ALT), aspartate transaminase (AST), liver aminopyrine $\mathrm{N}$-demethylase (AD), liver aniline hydroxylase (AH), liver 3,4-Methylenedioxyamphetamine (liver MDA), cytochrome P450 (P450), serum alkaline phosphatase (ALP), serum lactate dehydrogenase (LDH), cytochrome b5 (Cyto b5), glutathione reductase (GR), Liver glutathione $S$-transferase (GST), liver glutamyltransferase (GT), Liver $(\gamma$-GCS), serum liver 3,4-Methylenedioxyamphetamine

Table 1. Combined MDs and Homogeneity test in fixed effect model and random effect model. ${ }^{\text {a) }}$

\begin{tabular}{|c|c|c|c|c|c|}
\hline & \multicolumn{2}{|c|}{ Fixed effect model ${ }^{\mathrm{a})}$} & \multirow{2}{*}{$\frac{\text { Heterogeneity }^{\mathrm{b})}}{\text { p-value }}$} & \multicolumn{2}{|c|}{ Random effect model ${ }^{\text {a) }}$} \\
\hline & estimate & p-value & & estimate & $\mathrm{p}$-value \\
\hline ALT & -19.6989 & $<0.0001$ & $<0.0001$ & $-22.2402^{c)}$ & $<0.0001$ \\
\hline AST & -35.0759 & $<0.0001$ & $<0.0001$ & $-63.0643^{c)}$ & $<0.0001$ \\
\hline ALP & $-19.9995^{\mathfrak{c})}$ & $<0.0001$ & 0.2450 & -19.3157 & $<0.0001$ \\
\hline LDH & -10.2558 & $<0.0001$ & 0.0004 & $-12.4119^{c)}$ & $<0.0001$ \\
\hline Serum MDA & -0.2510 & 0.5857 & 0.0246 & -19.0803 & 0.1389 \\
\hline $\mathrm{SDH}$ & -21.6129 & $<0.0001$ & 0.0060 & $-23.2156^{\mathrm{c})}$ & $<0.0001$ \\
\hline $\mathrm{TP}$ & $0.4750^{c)}$ & 0.0014 & 0.5316 & 0.4750 & 0.0014 \\
\hline$\gamma$-GT & -21.6690 & $<0.0001$ & $<0.0001$ & $\left.-20.7797^{c}\right)$ & $<0.0001$ \\
\hline $\mathrm{AD}$ & -1.0339 & $<0.0001$ & $<0.0001$ & $-1.1376^{\mathrm{c})}$ & 0.0088 \\
\hline $\mathrm{AH}$ & -0.1734 & $<0.0001$ & $<0.0001$ & $-0.3022^{\mathrm{c})}$ & 0.0446 \\
\hline Liver MDA & -21.0995 & $<0.0001$ & $<0.0001$ & $-25.7005^{c)}$ & $<0.0001$ \\
\hline P450 & -0.2632 & $<0.0001$ & $<0.0001$ & $-0.3919^{c)}$ & 0.0049 \\
\hline Cyto b5 & -0.2256 & $<0.0001$ & $<0.0001$ & $-0.0852^{\mathrm{c})}$ & 0.0136 \\
\hline GR & 8.1787 & $<0.0001$ & $<0.0001$ & $8.3500^{c)}$ & $<0.0001$ \\
\hline GST & 49.2603 & $<0.0001$ & $<0.0001$ & $48.0553^{c)}$ & $<0.0001$ \\
\hline GT & 2.0149 & $<0.0001$ & $<0.0001$ & $1.9541^{\mathrm{c})}$ & $<0.0001$ \\
\hline$\gamma$-GCS & 0.7705 & $<0.0001$ & 0.0456 & $0.6966^{\mathrm{c})}$ & 0.0128 \\
\hline
\end{tabular}

a) The data were expressed the combined mean difference between the RH and the RHG about factors and p-value for testing that the mean difference is not significantly different with 0 .

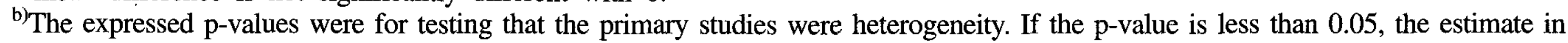
random effect model is recommended

c) The mean of the RH for factor was significantly different form the RHG (p-value<0.05), thus the anti-hepatotoxic effect of ginseng were significant. 
(serum MDA), serum sorbitol dehydrogenase (SDH), serum total protein (TP), serum $\gamma$-glutamyltransferase $(\gamma$-GT). The primary studies were listed in reference from 10 to 19 . The unit of each factor was uniformly changed.

\section{Measure and models for combining}

Since the outcome measure of factors was continuous type, the mean differences (MD) between the RH and RHG about them were used as the association means, and combined as indication of the effect of ginseng. The inverse variance method was used in two models; the fixed effect model and the random effect model. In the study of the fixed effect model, it is assumed that there is no heterogeneity between the results of studies and the effect measured in the study population has a single value. On the other hand, the studies of the random effect model are heterogeneous and it includes two sources of variation; variation between two studies or variation within the study. If the homogeneity assumption is plausible by a heterogeneity test, the effect size is estimated by using a variation within the study in the fixed effect model. If not, a variation between studies is estimated and included in a total variation. The fixed effect estimates and the random effect estimates were presented in Table 1.

\section{Identification the statistical bias}

Publication bias was assessed by using a funnel plot method. The calculated association measures, the MDs in our study, of studies about the factor are plotted against the inverse of the estimated standard error (SE) of them. The results from smaller studies will be more widely spread around the mean effect because of larger random error. If there is no publication bias, the shape of plot is funnel or cone around the mean. To test the asymmetry of a funnel plot, Egger et al.(1997) suggested a linear regression test based on a regression analysis of Galbraith's radial plot, Garbraith's radial plots plot the standardized effect sizes of outcome from each study against the inverse of standard error. The intercept of simple regression of them is used to measure asymmetry. If the estimated intercept is significantly different from 0 , then there may be publication bias. A positive intercept indicates that more studies are associated with bigger effect. Results of Egger's linear regression test are presented in Table 2 about factors. The trim and fill method provides estimate combined effect sizes by 2 steps process, in which the asymmetric data is trimmed and estimated. The trim and fill method is used to compare the original estimates.
Table 2. Estimated intercepts and p-values of Egger's linear regression test.

\begin{tabular}{l|rc}
\hline \hline & \multicolumn{2}{|c}{ Egger's linear regression } \\
\cline { 2 - 3 } & Intercept $^{\mathrm{a})}$ & p-value $^{\mathrm{b})}$ \\
\hline ALT & -0.5426 & 0.5152 \\
ALP & -3.4823 & 0.1568 \\
LDH & -0.6026 & 0.5730 \\
Serum MDA & -0.8864 & 0.3606 \\
SDH & -1.5960 & 0.1188 \\
TP & -7.7006 & 0.2005 \\
$\gamma$-GT & 1.2785 & 0.4156 \\
AD & 1.8620 & 0.7543 \\
AH & -4.0415 & 0.5356 \\
Liver MDA & -8.7456 & 0.0659 \\
P450 & -2.4548 & 0.1309 \\
Cyto b5 & -6.0224 & 0.4394 \\
GR & 65.7270 & 0.0810 \\
GST & 8.6398 & 0.4892 \\
GT & 17.6729 & 0.2490 \\
$\gamma$-GCS & -6.3666 & 0.4393 \\
\hline
\end{tabular}

a) The data were expressed the intercept of Egger's linear regression about factors.

${ }^{b}$ The expressed $p$-values were for testing that the estimated intercept is not significantly different with 0 . If the p-value is less than 0.05 , the combined MD in Table 1 possibly exist the publication bias, and the additional analysis is required.

\section{Software used for the meta-analysis}

Version 1.5 of MIX program was used for combining MDs, plotting the funnel plots and checking the publication. The MIX program is a tool for performing metaanalysis, and distinguishably provides the extensive graphical outputs. It can be downloaded free of charge via http://www.mix-for-meta-analysis.info ${ }^{8)}$.

\section{Results}

In Table 1, there are the combined MDs by the inverse variance weighted method and p-values to test the hypothesis $\mathrm{H} 0: \mathrm{MD}=0$ in the fixed effect model and in random effect model with p-value about test the hypothesis $\mathrm{H} 0$ : Heterogeneity among primary studies. In Fig. 1, the trim and fill funnel plots of effect factors were presented. The intercepts and p-value of Egger's linear regression test were presented to checking the publication bias in Table 2 , and the fixed effect estimate and random effect estimate by trim and fill method were presented in Table 3 . 


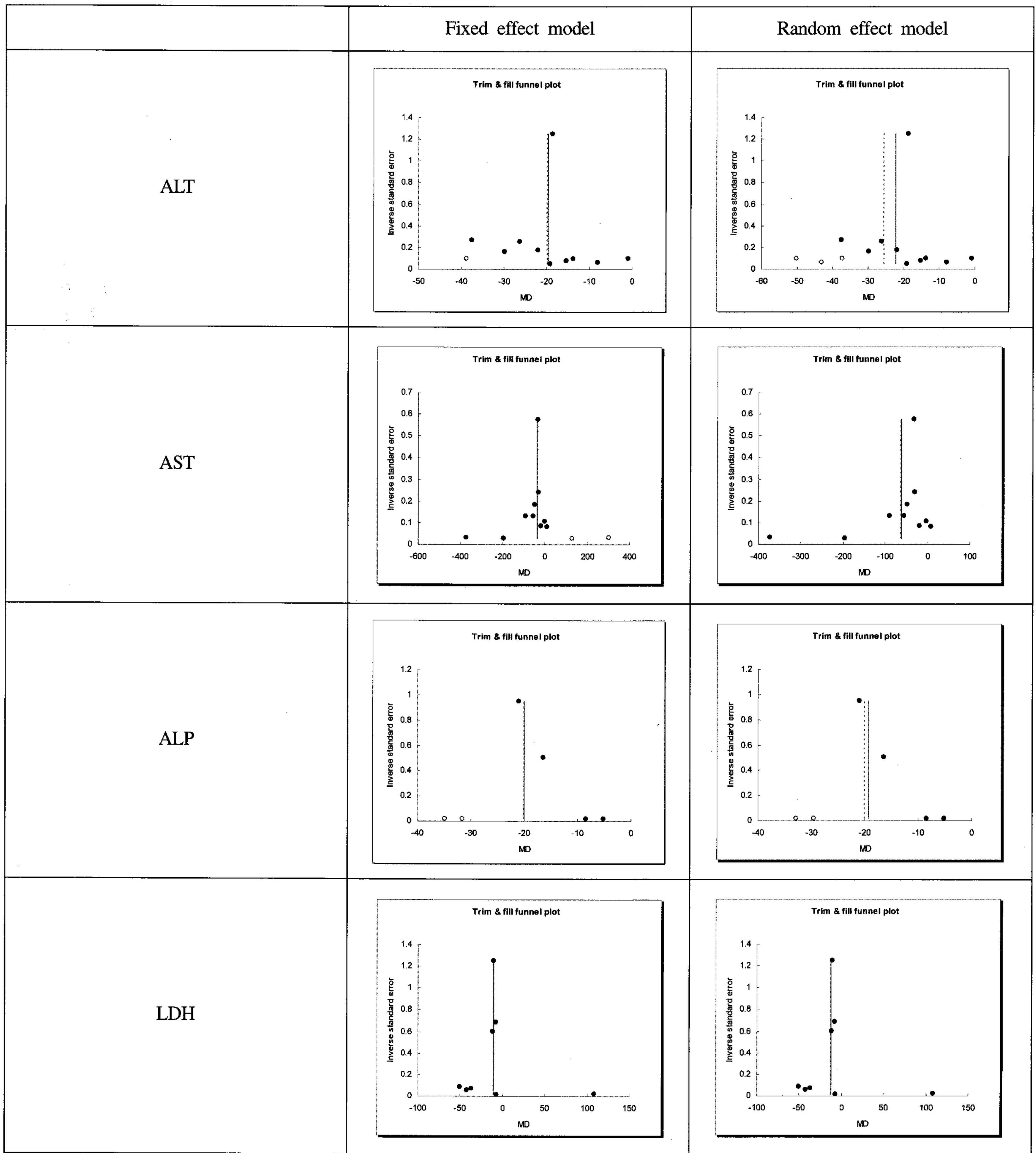

a) The data were plotted the mean difference between the RH and the RHG verse inverse standard error.

- Dots $(\bullet)$ are original data and circles $(\circ)$ are filled data allowing $95 \%$ confidence interval to be calculated for the adjusted pooled estimate. The results of original data were indicated by a solid line, and the result of the trim and fill method by a dotted line. If the shape of dots looks like symmetric funnel about line, then it is assumed that the combined estimate has no publication bias.

Fig. 1. Trim and fill funnel plot: In fixed effect model and random effect model about plasma factors ${ }^{\mathrm{a})}$ 


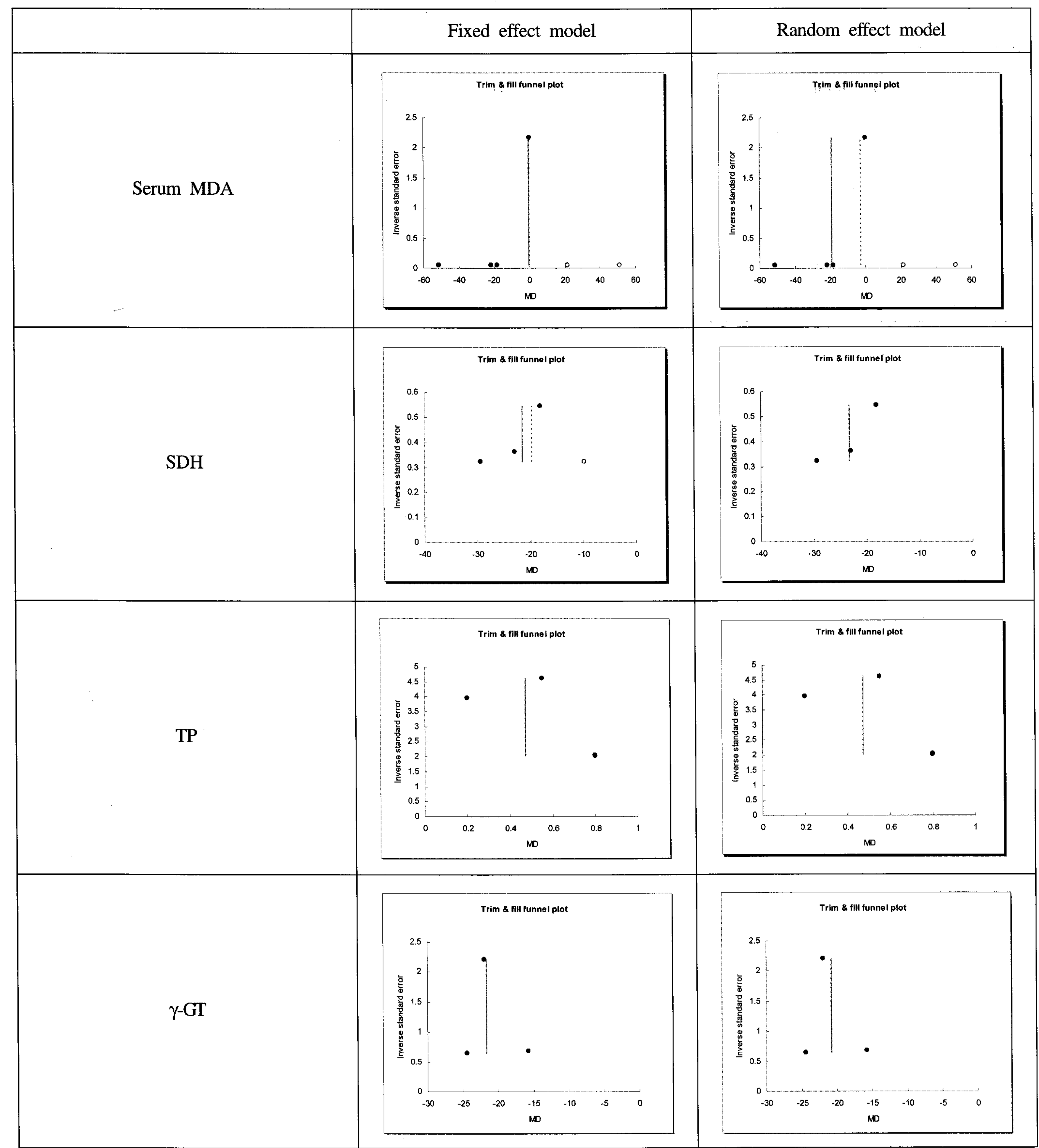

a) The data were plotted the mean difference between the RH and the RHG verse inverse standard error.

- Dots $(\bullet)$ are original data and circles $(\circ)$ are filled data allowing $95 \%$ confidence interval to be calculated for the adjusted pooled estimate. The results of original data were indicated by a solid line, and the result of the trim and fill method by a dotted line. If the shape of dots looks like symmetric funnel about line, then it is assumed that the combined estimate has no publication bias.

Fig. 1. Continued. 


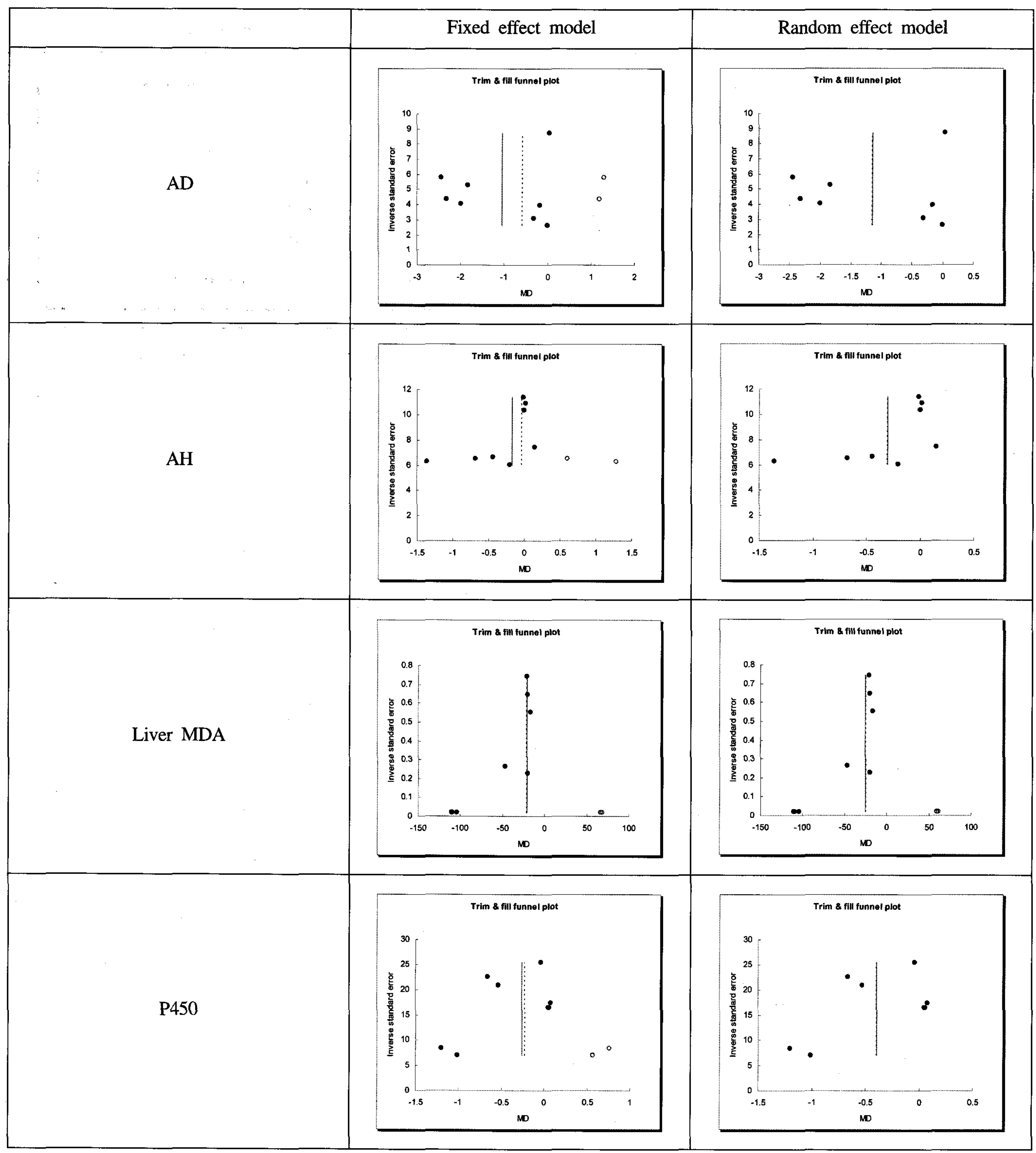

a) The data were plotted the mean difference between the RH and the RHG verse inverse standard error.

- Dots $(\bullet$ ) are original data and circles $(\circ)$ are filled data allowing $95 \%$ confidence interval to be calculated for the adjusted pooled estimate. The results of original data were indicated by a solid line, and the result of the trim and fill method by a dotted line. If the shape of dots looks like symmetric funnel about line, then it is assumed that the combined estimate has no publication bias.

Fig. 2. Trim and fill funnel plot: In fixed effect model and random effect model about enzyme factors. ${ }^{\text {a) }}$ 


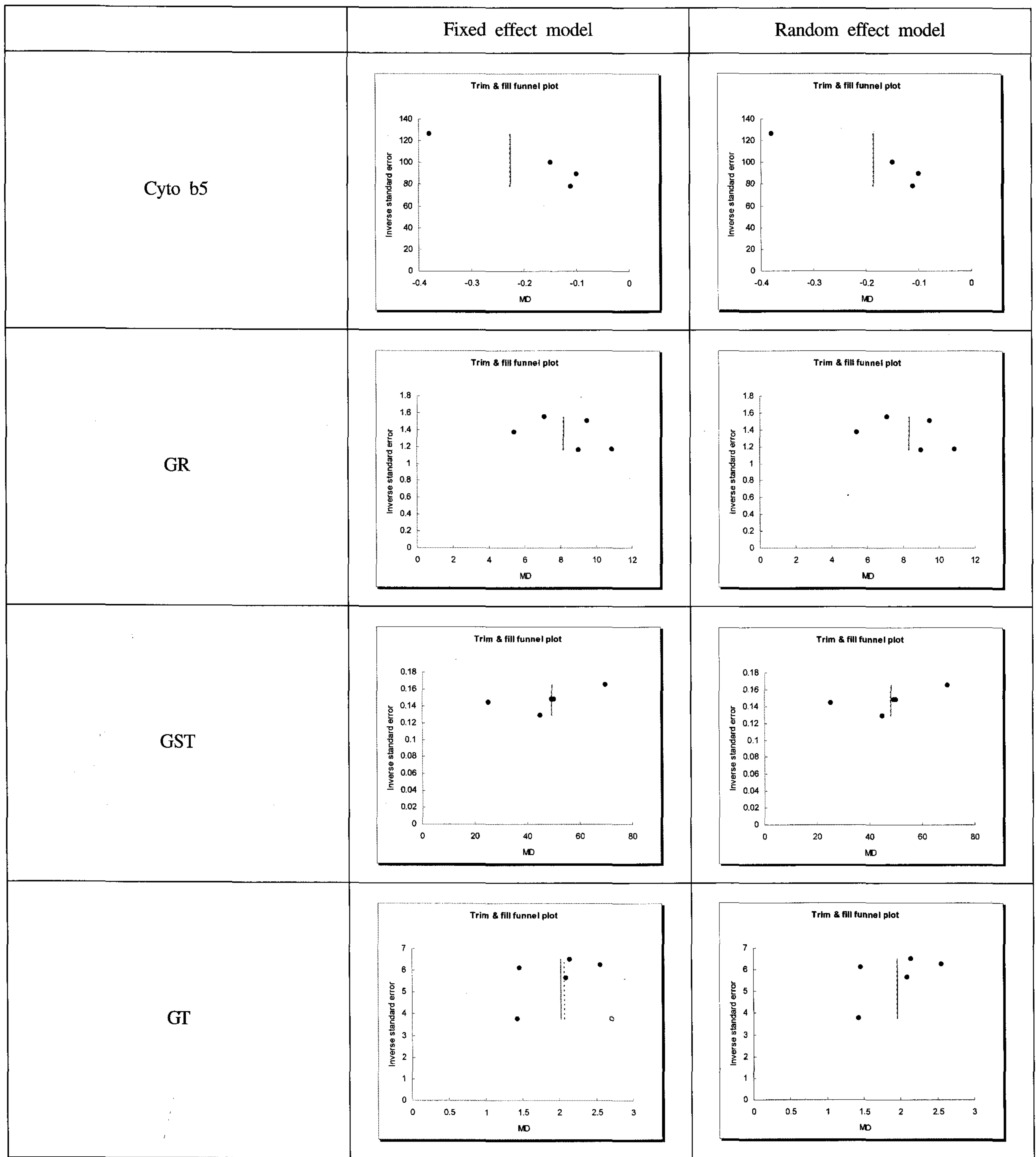

a) The data were plotted the mean difference between the RH and the RHG verse inverse standard error.

- Dots $(\bullet)$ are original data and circles $(\circ)$ are filled data allowing $95 \%$ confidence interval to be calculated for the adjusted pooled estimate. The results of original data were indicated by a solid line, and the result of the trim and fill method by a dotted line. If the shape of dots looks like symmetric funnel about line, then it is assumed that the combined estimate has no publication bias.

Fig. 2. Continued-1. 


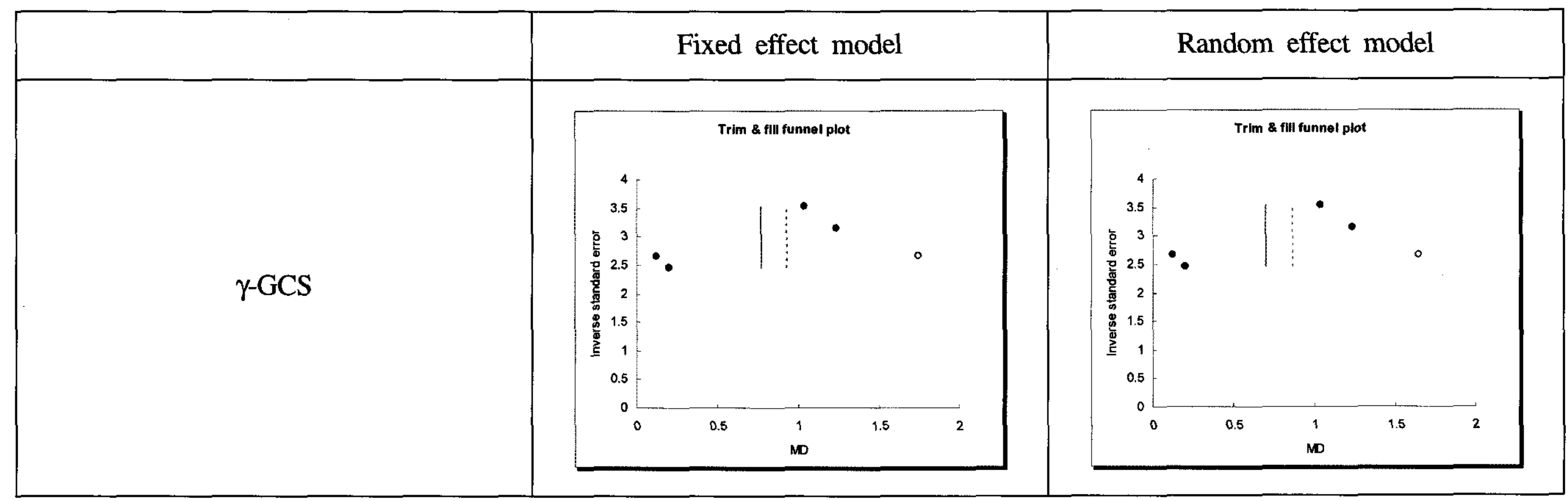

a) The data were plotted the mean difference between the RH and the RHG verse inverse standard error.

- Dots $(\bullet)$ are original data and circles $(\circ)$ are filled data allowing $95 \%$ confidence interval to be calculated for the adjusted pooled estimate. The results of original data were indicated by a solid line, and the result of the trim and fill method by a dotted line. If the shape of dots looks like symmetric funnel about line, then it is assumed that the combined estimate has no publication bias.

Fig. 2. Continued-2.

\section{Means Difference in plasma factors}

The considered plasma factors were ALT, AST, ALP, LDH, serum MDA, SDH, TP and $\gamma$-GT. In fixed effect model, the effect of ginseng to serum MDA was not significant $(0.5857)$, but the others factor were significant $(<0.001)$. The $p$-values of heterogeneity test among studies about ALP and TP were more than 0.05, but the others were less. In random effect model, the effect of ginseng to serum MDA was not significant, and the others were significant $(<0.01)$.

The mean differences of ALT, AST, LDH, serum MDA, SDH and $\gamma$-GT between RH and RHG were estimated by random effect model because of heterogeneity among studies, and those of ALP and TP were by fixed effect model. By upper reasons mentioned, the level of ALT, AST, ALP, LDH, SDH and $\gamma$-GT in plasma were significantly reduced $(<0.01)$, and the level of TP in plasma was increased by treatment with ginseng $(<0.01)$. The mean of serum MDA in RH was not significantly different with them of RHG (0.1389).

\section{Means Difference in enzyme factors}

The considered enzyme factors were $\mathrm{AD}, \mathrm{AH}$, liver MDA, P450, Cyto b5, GR, GST, GT and $\gamma$-GCS. In fixed effect model, the effect of ginseng to all enzyme factors were strongly significant $(<0.001)$. The p-value of heterogeneity test among studies about $\gamma$-GCS was less than 0.05 , and the others were less than 0.01 . In random effect model, the effect of ginseng to all considered-enzyme factors was significant $(<0.05)$.
The mean differences of were all considered enzyme factors, AD, AH, liver MDA, P450, Cyto b5, GR, GST, GT and $\gamma$-GCS, between RH and RHG were estimated by random effect model because of heterogeneity among studies. By reasons mentioned, the level of $\mathrm{AD}, \mathrm{AH}$, liver MDA, P450 and Cyto b5 were significantly reduced $(<0.05)$, and the level of GR, GST, GT and $\gamma$-GCS were increased by treatment with ginseng $(<0.05)$. Especially, the mean of $\mathrm{AD}$, liver MDA, P450, GR, GST and GT were very significantly different with them of RHG $(<0.01)$.

\section{Publication Biases}

For checking the existence of publication bias in estimated MDs in Table 1, the funnel plot and Egger's linear regression plot was used. Because the dotted plots in funnel plot about them did not look like funnel or cone, the estimates in Table 1 probably have the publication bias about some factors, ALP, LDH, SDH, AH, liver MDA, cyto b5, GST, GT and $\gamma$-GCS. To checking the symmetry of the data in funnel plot, Egger's linear regression test was used. Because the intercept of Egger's linear regression test were not significantly different with 0 , the symmetry of data was plausible.

The estimates by trim and fill method in Table 3 are calculated after trimming asymmetric data and filling by imputation on the assumption that the publication bias in estimates probably exist. By trim and fill method about plasma factors, the $95 \%$ confidence interval of mean difference about ALP and TP did not include 0 in fixed 
Table 3. $95 \%$ confidence intervals of combined MDs by trim and fill method. ${ }^{\text {a) }}$

\begin{tabular}{|c|c|c|c|c|c|c|}
\hline & \multicolumn{3}{|c|}{ Fixed effect estimate } & \multicolumn{3}{|c|}{ Random effect estimate } \\
\hline & T-F est & CI_low & CI_up & T-F est & CI_low & CI_up \\
\hline ALT & -19.8037 & -21.2622 & -16.8867 & -25.4755 & -32.1837 & -12.0591 \\
\hline AST & -34.0258 & -36.8270 & -28.4234 & -34.0258 & -36.8270 & -28.4234 \\
\hline ALP & -20.0057 & -21.8207 & -16.3757 & -20.0048 & -21.8198 & -16.3748 \\
\hline LDH & -10.2558 & -11.5147 & -8.9969 & -12.4119 & -16.9530 & -7.8708 \\
\hline Serum MDA & -0.2081 & -1.1086 & 1.5929 & -2.6674 & -25.1192 & 19.7844 \\
\hline $\mathrm{SDH}$ & -19.6719 & -22.1277 & -14.7603 & -23.2156 & -29.7001 & -16.7311 \\
\hline $\mathrm{TP}$ & 0.4750 & 0.1844 & 0.7656 & 0.4750 & 0.1844 & 0.7656 \\
\hline$\gamma$-GT & -21.6690 & -22.4835 & -20.8545 & -20.7797 & -24.8316 & -16.7277 \\
\hline $\mathrm{AD}$ & -0.5654 & -0.6875 & -0.3212 & -0.5654 & -0.6875 & -0.3212 \\
\hline $\mathrm{AH}$ & -0.0352 & -0.1118 & 0.0476 & -0.0352 & -0.1118 & 0.0476 \\
\hline Liver MDA & -21.0496 & -22.7101 & -17.7286 & -24.9158 & -32.0569 & -10.6336 \\
\hline P450 & -0.2209 & -0.2588 & -0.1451 & -0.2209 & -0.2588 & -0.1451 \\
\hline Cyto b5 & -0.2256 & -0.2353 & -0.2158 & -0.1852 & -0.3322 & -0.0381 \\
\hline GR & 8.1788 & 7.5364 & 8.8211 & 8.3500 & 6.5035 & 10.1965 \\
\hline GST & 49.2604 & 43.3121 & 55.2086 & 48.0553 & 33.5581 & 62.5524 \\
\hline GT & 2.0698 & 1.9234 & 2.3626 & 1.9541 & 1.5363 & 2.3719 \\
\hline$\gamma$-GCS & 0.9315 & 0.6307 & 1.5331 & 0.8659 & 0.3316 & 1.4001 \\
\hline
\end{tabular}

a) The estimates were calculated by the trim-and fill method. T-F est., CI_low and CI_up are the trim-and fill estimate, lower bound and upper bound of $95 \%$ confidence interval respectively.

b) If the 0 was not included between CI_low and CI_up, the mean of the RH for factor was significantly different form the RHG (p-value $<0.05$ ), thus the anti- hepatotoxic effect of ginseng were significant.

effect model, them about ALT, AST, LDH, SDH and $\gamma$-GT did not include 0 in random effect model, but about serum MDA did include 0. By trim and fill method about enzyme factors, the $95 \%$ confidence interval of mean difference about AD liver MDA, P450, Cyto b5, GR, GST, GT and $\gamma$-GCS did not include 0 in random effect model, but about AH did include 0 .

Although the publication biases of estimated MDs between RH and RHG about considered factors are doubtable by funnel plot, the ginseng significantly effected to ALT, AST, ALP, LDH, SDH, TP and $\gamma$-GT, AD, liver MDA, P450, Cyto b5, GR, GST, GT and $\gamma$-GCS except serum MDA and $\mathrm{AH}$.

\section{CONCLUSION}

This study investigated the anti-hepatotoxity of ginseng about the level of ALT, AST, ALP, LDH, serum MDA, SDH, TP and $\gamma$-GT in plasma factors, and about the level of AD, AH, liver MDA, P450, Cyto b5, GR, GST, GT and $\gamma$-GCS in enzyme factors by test which the mean differences, MD, between RH and RHG about them were significantly different with 0 in fixed effect model or in random effect model. The adjusted estimates of MDs by trim and fill method were significantly different with 0 in ALT, AST, ALP, LDH, SDH, TP, and $\gamma$-GT in plasma factors and AD, liver MDA, P450, Cyto b5, GR, GST, GT and $\gamma$-GCS in enzyme factors $(<0.05)$. But, the effect to serum MDA and AH were not significant $(>0.05)$.

\section{ACKNOWLEDGMENT}

This work was supported by the Korea Research Foundation Grant funded by the Korean Government (MOEHRD). (KRF-2005-005-J13001).

\section{REFERENCE}

1. Lee, W. M. : Drug-induced hepatotoxicity. $N$ Engl J Med. 349, 474-485 (2003).

2. Nilesh Mehta, and Lisa Ozick. : Drug-Induced Hepatotoxicity. http://www.emedicine.com/Med/topic3718.htm

3. Cupp, M. J. : Herbal remedies: adverse effects and drug interactions. Am Fam Physician 59, 1239-1244 (1999).

4. De Smet PAGM, Keller, K., Hansel, R. and Chandler, R. F. eds. : Adverse effects of herbal drugs. Berlin, Germany: Springer - Verlag (1997).

5. Kiefer, D. and Pantuso, T. : Panax ginseng. American Acad- 
emy of Family Physicians 68, 1539-1542 (2003).

6. Health Organization. WHO monographs on selected medicinal plants. Geneva: World Health Organization (1999).

7. Han, K., Shin, I. C., Choi, K. J., Yun, Y. P., Hong, J. T. and Oh, K. W. : Korea red ginseng water extract increases nitric oxide concentrations in exhaled breath. Nitric Oxide. 2005 May; 12(3), 159-162.

8. Choi, K. and Kook, S. : What is the meta-analysis?. Metaanalysis using program MIX. FreeAcademy, Seoul, Korea. pp. 2-4 (2007)

9. Sutton, A., Abrams, K., Jones, D., Sheldon, T. and Song, F. : Introduction, Publication bias. In Methods for Meta-Analysis in Medical Research. Wiley. pp. 3-13, 117-119 (2000).

10. Kim, S. Y., Kim, Y. C., Byun, S. J., Kim, E. : The effect of Ginsenosides on Galactosamine-induced Hepatotxicity, Kor. J. Pharmacogn., 22(4), 219-224 (1991).

11. Kwak, Y. S., Kyung, J. S., Song, Y. B., Wee, J. J., Prak, J. D. : Effect of Crude saponine from Red-ginseng effluex on Blood biochemical parameters in Rats Acutely Exposed to 2,3,7,8tetrachlorodibenzo-p-dioxin(TCDD), J. Ginseng Res., 30(1), 8-14 (2006).

12. Lee, J. K., Han, Y. N., Kim, N. Y., Choi, J. W. : The Therapeutic Effects of Korean Red Ginseng on Carbon Tetrachloride and Galactosamine-induced Hepatotoxicity in Rats, $J$. Ginseng Res., 27(1), 11-16 (2003).

13. Lee, J. K., Kim, N. Y., Han, Y. N. and Choi, J. W. : Effects of Pretreated Korean Red Ginseng on Carbon Tetrachloride and Galactosamine-induceed Hepatotoxicity in Rats, J. Ginseng
Res., 27(1), 1-10 (2003).

14. Lee, J. K., Choi, J. W., Kim, S. H., Kim, H. K. and Han, Y. N. : Biological Activities of Acidic Polysaccharide of Korean Red Ginseng.III.-Effects on Metabolizing Activities in Acetaminophen-treated Rats, J. Ginseng Res., 22(4), 267-273 (1998).

15. Wee, J. J., Heo, J. N., Kim, M. W. and Kang, D. Y. : Protective Effect of Korean Red Ginseng against Oxidative Damage by Carbon Tetrachloride in Rat, Korean J. Ginseng Sci., 20(2), 154-158 (1996).

16. Song, K. H., Kim, D. H., Choi, K. J. : The effect of Aquaacupuncture of Total Saponin on the Damaged Liver Induced by Carbon Tetrachloride in Rats, Korean J. Vet Clin Med, 13(2), 108-113 (1996).

17. Kim, H. Y., Lee, Y. H. and Kim, S. I. : Antihepatotoxic Components of Korean Ginseng: Effect on Lipid Peroxidation, Korean Biochem. J., 22(1), 12-18 (1989).

18. Kim, Y. S. : Protective Effect of Ginseng Polysacchride Fraction on $\mathrm{CCl}_{4}$-induced Hepatotoxcity in vitro and in vivo, Korean J. Ginseng Sci., 19(2), 108-113 (1995).

19. Ha, B. J. : Effects of Rhynchosia molubilis Saponine on Hpatotoxicity and Pathology, Journal of Life Science, 16(2), 186191 (2006).

20. Sutton, A., Abrams, K., Jones, D., Sheldon, T. and Song, F. : Methods for Meta-Analysis in Medical Research. Wiley (2000).

21. Whiteead, A. Meta- Analysis of Controlled Clinical Trials. John Wiley \& Sons (2002). 